\title{
MAJORIZATION REFINEMENTS OF KY FAN'S EIGENVALUE INEQUALITY AND RELATED RESULTS
}

\author{
MAREK NIEZGODA
}

Abstract. In this note we show the anti-isotonicity of a certain operator function induced by the eigenvalue map $\lambda(\cdot)$ on the space of Hermitian matrices. In consequence, we obtain some majorization refinements of the Ky Fan's eigenvalue inequality. Thus we extend Maligranda's inequalities from a norm to the eigenvalue map, and from the usual order on $\mathbb{R}$ to the majorization preorder on $\mathbb{R}^{n}$.

Mathematics subject classification (2020): 15A42, 15A18, 15A23, 06F20.

Keywords and phrases: Maligranda's inequalities for norms, majorization, eigenvalues, Ky Fan's eigenvalue inequality, convex cone, simultaneously diagonalizable (SD) matrices.

\section{REFERENCES}

[1] R. Bhatia, Matrix Analysis, Graduate Texts in Mathematics 169, Springer-Verlag, New York, 1997.

[2] J. A. Clarkson, Uniformly convex spaces, Trans. Amer. Math. Soc., 40, (1936), 396-414.

[3] C. F. Dunkl and K. S. Williams, A simple norm inequality, Amer. Math. Monthly, 71, (1964), 53-54.

[4] K. FAn, On a theorem of Weyl concerning eigenvalues of linear transformations I, Proc. Nat. Acad. Sci. U.S.A., 35, (1949), 652-655.

[5] A. S. LEWIS, Group invariance and convex matrix analysis, SIAM J. Matrix Anal. Appl., 17, (1996), 927-949.

[6] V. B. LIDSKII, On the characteristic numbers of the sum and product of symmetric matrices, Doklady Akad. Nauk SSSR (N.S.), 75, (1950), 769-772.

[7] L. Maligranda, Simple norm inequalities, Amer. Math. Monthly, 113, 3 (2006), 256-260.

[8] A. W. Marshall, I. Olkin and B. C. Arnold, Inequalities: Theory of Majorization and Its Applications, 2nd Ed., Springer Series in Statistics, Springer, New York, 2011.

[9] J. L. MASSERA AND J. J. SCHÖFFER, Linear differential equations and functional analysis I, Ann. Math., 67, (1958), 517-573.

[10] P. R. MERCER, The Dunkl-Williams inequality in an inner product space, Math. Inequal. Appl., 10, 2 (2007), 447-450.

[11] M. NiezGodA, On Maligranda like inequalities for G-majorization, Linear Multilinear Alg., (2021), (to appear).

[12] H. Wielandt, An extremum property of sums of eigenvalues, Proc. Amer. Math. Soc., 6, (1955), $106-110$.

[13] Z.-F. ZUO, On James type constants and the normal structure in Banach spaces, Math. Inequal. Appl., 23, 1 (2020), 341-350. 